\title{
Uso de contraceptivos e fatores associados entre adolescentes de 15 a 18 anos de idade em Unidade de Saúde da Família
}

\author{
Contraceptive use and associated factors among adolescents from 15 to 18 years \\ old in a Family Health Unit
}

\author{
Marcela Zanini' ${ }^{1}$, Joana D. S. Selvante ${ }^{2}$, Fabio F. Quagliato ${ }^{3}$
}

Zanini M, Selvante JDS, Quagliato FF. Uso de contraceptivos e fatores associados entre adolescentes de 15 a 18 anos de idade em Unidade de Saúde da Família / Contraceptive use and associated factors among adolescents from 15 to 18 years old in a Family Health Unit. Rev Med (São Paulo). 2017 jan.-mar.;96(1):32-4.

RESUMO: O início da atividade sexual em mulheres jovens pode causar uma série de consequências devido ao uso inapropriado ou à falta de acesso aos métodos anticoncepcionais. Considerando aquelas que fazem uso de contraceptivos, em muitos casos o uso é incorreto ou inconsistente. O objetivo deste estudo é avaliar o conhecimento das mulheres de 15 a 18 anos sobre diferentes métodos contraceptivos, por meio de questionário. 25 mulheres foram entrevistadas e $55,52 \%$ relataram ter sido sexualmente ativas, das quais $44 \%$ relataram que usam regularmente algum tipo de método contraceptivo. O preservativo masculino é o método mais conhecido (84\%) em todas as idades. A fonte de informação mais citada em relação a cada idade foi a família (15 e 16 anos), a escola (17 anos) e profissionais de saúde (18 anos). $36 \%$ das entrevistadas admitiram nunca ter ido a uma consulta com um ginecologista ou o médico de família. Concluímos que, mesmo tendo acesso a diversos meios de informação, as jovens não adotam uma vida sexual segura, deixando de usar ou usando de forma inadequada os diversos métodos anticoncepcionais disponíveis.

Descritores: Anticoncepção; Adolescente; Gravidez.

\begin{abstract}
The initiation of sexual activity in young women can cause a number of consequences due to inappropriate use or the lack of access to contraceptive methods. Among those who make use of contraceptives, in many cases the use is incorrect or inconsistent. The aim of this study is to evaluate the knowledge of women from 15 to 18 years old of different contraceptive methods using a questionnaire. Twenty five women were interviewed and $55.52 \%$ of them reported being sexually active. Among the sexual active women, $44 \%$ confirmed that they regularly use some kind of contraceptive method. The male condom is the most known method (84\%) at all ages. The most cited source of information in relation to each age were the family among the girls between 15 and 16 years old, the school (17 years old) and health professional by 18 years old girls. $36 \%$ of respondents admitted they had never been to a consultation with a gynecologist or the family doctor. We conclude that even having access to different means of information, young women do not adopt a safe sex life, not using or using in the wrong way the various contraceptive methods available.
\end{abstract}

Keywords: Contraception; Adolescent; Pregnancy.

Apresentação Oral no BRAINCOMS - Brazilian International Congress of Medical Students, Universidade Federal de São Paulo (Escola Paulista de Medicina), São Paulo - SP, 20-22 out. 2016. Área de conhecimento (Tabela CNPq): Medicina Preventiva - 4.06.03.00-8.

1. Graduanda em Medicina, Centro Universitário Barão de Mauá, Ribeirão Preto. E-mail: ma219@hotmail.com.

2. Graduanda em Medicina, Centro Universitário Barão de Mauá, Ribeirão Preto. E-mail; joanasselvante@gmail.com.

3. Docente de Saúde Coletiva, Centro Universitário Barão de Mauá. E-mail: fabio.franchi@baraodemaua.br.

Endereço para correspondência: Marcela Zanini. Rua Treze de Maio, 110. Bairro São Domingos. CEP: 13471030 - Americana, São Paulo.E-mail:ma219@hotmail.com. 


\section{INTRODUÇÃO}

Operíodo da adolescência é uma fase de psicológicas ${ }^{1,2,3,4}$. O indivíduo em crescimento está numa fase de desenvolvimento de características sexuais adultas e, nesse contexto, começam as primeiras experiências sexuais $^{1,2}$. A iniciação da vida sexual nessa faixa etária pode trazer várias consequências pelo uso inadequado ou pelo desconhecimento de métodos contraceptivos, dentre elas a gravidez precoce/indesejada ${ }^{4,5,6,7}$. Devido aos riscos relacionados à gravidez na adolescência, esse grupo requer uma maior atenção dos profissionais da área da saúde ${ }^{1,3}$.

Dentre as adolescentes que engravidaram cedo, identifica-se uma tendência ao não uso dos métodos contraceptivos $^{1,8,9}$. Muitas argumentam que não conhecem os métodos ou que não acreditam que possam engravidar; outras dizem que o parceiro não gosta de usar ou até mesmo que não têm condições para comprar ${ }^{5,9}$. Levando em conta as que fazem uso dos contraceptivos, em muitos casos, elas não os utilizam corretamente ou se esquecem do uso consistente, deixando nítido o risco a que esse grupo está exposto ${ }^{4,5,8,9,10}$. Desse modo, a realidade dessas jovens pode ser modificada, quando há um acesso a informações confiáveis e um uso adequado dos contraceptivos, evitando situações de risco $^{3,11}$.

\section{OBJETIVO}

O objetivo deste estudo é avaliar o conhecimento das mulheres de 15 a 18 anos sobre diferentes métodos contraceptivos, por meio de questionário.

\section{CAUSUÍSTICA E MÉTODO}

Foi realizado um estudo descritivo, com aplicação de um questionário de 25 questões para mulheres de 15 a 18 anos, atendidas em uma Unidade de Saúde da Família do município de Ribeirão Preto - SP.

\section{RESULTADOS E DISCUSSÃO}

Como podemos observar na Tabela 1, os métodos mais conhecidos pelas entrevistadas foram a camisinha masculina e o anticoncepcional oral combinado, métodos disponíveis na rede pública de saúde, ou seja, de fácil acesso pela população. 55,52\% relataram ser sexualmente ativas, contudo, 66\% dessas negaram o uso regular de algum método contraceptivo e apenas $13,95 \%$ disseram que o parceiro fez uso do método nos últimos 12 meses. Isso mostra que elas conhecem os métodos, uma vez que 92\% das entrevistadas referem conhecer pelo menos um tipo de método contraceptivo, mas não fazem uso regular ou adequado, apesar do fácil acesso a eles. Essa pequena parcela de parceiros que utilizaram o método nos últimos
12 meses mostra a baixa adesão da população masculina aos métodos de barreira, que além de deixá-los expostos a infecções sexualmente transmissíveis, aumenta o risco de uma gravidez não planejada.

Tabela 1. Métodos anticoncepcionais sobre os quais as entrevistadas têm conhecimento

\begin{tabular}{lcc}
\hline Métodos anticoncepcionais que conhece & $\mathbf{N}^{\circ}$ & Porcentagem \\
\hline Camisinha Masculina & 21 & $84 \%$ \\
Anticoncepcional Oral Combinado & 18 & $72 \%$ \\
Anticoncepcional Injetável & 13 & $52 \%$ \\
Camisinha Feminina & 12 & $28 \%$ \\
Vasectomia & 8 & $32 \%$ \\
Dispositivo Intra Uterino & 6 & $24 \%$ \\
Ligadura de Trompas & 4 & $16 \%$ \\
Diafragma & 4 & $16 \%$ \\
Tabelinha & 1 & $4 \%$ \\
Coito Interrompido & 1 & $4 \%$ \\
Espermaticida & 1 & $4 \%$ \\
\hline
\end{tabular}

Das pacientes que já tiveram sua primeira relação sexual, $30,47 \%$ relataram ter parceiro fixo e $41,18 \%$ não usaram preservativo em sua última relação. Diante desses dados podemos observar que uma grande parcela das entrevistadas não possui parceiro fixo e não têm o hábito de usar preservativo em suas relações, mais uma vez reforçando o comportamento de risco adotado por essa população. Isso explica, em parte, a epidemia de infecções sexualmente transmissíveis enfrentada atualmente, uma realidade preocupante, que precisa de intervenção, sendo a educação em saúde uma estratégia eficaz para isso.

Das entrevistadas, 43,3\% já fizeram uso de contraceptivo de emergência (pílula do dia seguinte), $10,23 \%$ já tiveram uma ou mais gestações prévias e $4,65 \%$ referem episódio de aborto anterior. O uso inadequado dos contraceptivos pode ser uma das causas das gravidezes indesejadas no grupo estudado, pois, por falta de conhecimento ou pelo uso aparentemente mais fácil, a pílula do dia seguinte é usada rotineiramente como forma de contracepção. A frequência do uso desse tipo de método não está especificada no questionário, mas se as entrevistadas estão usando a pílula do dia seguinte como único meio de contracepção de maneira recorrente, há um maior risco de falha do método, já que esse só deve ser usado em situações específicas e não de maneira rotineira.

A Tabela 2 mostra os meios de informação que as entrevistadas relatam usar para obter conhecimento sobre sexo e contracepção. Mesmo mencionando a procura por profissionais de saúde como forma de obter informação sobre contracepção, as jovens não têm uma vida sexual segura, como identificado anteriormente. 
Tabela 2. Agentes de informação referidos pelas entrevistadas

\begin{tabular}{lcc}
\hline Agentes de Informação & $\mathbf{N}^{\mathbf{0}}$ & Porcentagem \\
\hline Familiares & 14 & $56 \%$ \\
Profissionais da Saúde & 10 & $40 \%$ \\
Escola & 10 & $40 \%$ \\
Amigos & 6 & $24 \%$ \\
Parceiro & 6 & $24 \%$ \\
TV, Internet e Rádio & 5 & $20 \%$ \\
Jornais, Revistas e Folhetos & 3 & $12 \%$ \\
Nenhum & 3 & $12 \%$ \\
Outros & 0 & 0 \\
\hline
\end{tabular}

\section{REFERÊNCIAS:}

1. Arcanjo CM, Oliveira MIV, Bezerra MGA. Gravidez ehm adolescentes de uma unidade municipal de saúde em fortaleza - Ceará. Esc Anna Nery Rev Enferm. 2007;11(3):44551. Disponível em: http://www.scielo.br/pdf/ean/v1ln3/ v11n3a08.

2. Lourenço B, Queiroz LB, Da Silva LEV, Leal MM. Medicina de adolescentes. 25a ed. São Paulo: Manole; 2015.

3. Martins LBM, Costa-Paiva L, Osis MJD, De Sousa MH, Neto AMP, Tadini V. Conhecimento sobre métodos anticoncepcionais por estudantes adolescentes. Rev Saude Publica. 2006;40(1):57-64. Disponível em: http://www.scielo. $\mathrm{br} / \mathrm{pdf} / \mathrm{rsp} / \mathrm{v} 40 \mathrm{n} 1 / 27116 . \mathrm{pdf}$

4. Yazlle MEHD. Gravidez na adolescência. Rev Bras Ginecol Obstetr. 2006;28(8):443-5. http://dx.doi.org/10.1590/S010072032006000800001 .

5. Gama SGN, Szwarcwald CL, Leal MC, Theme Filha MM. Gravidez na adolescência como fator de risco para baixo peso ao nascer no Município do Rio de Janeiro, 1996 a 1998. Rev Saude Publica. 2001;35(1):74-80. http://dx.doi.org/10.1590/ S0034-89102001000100011.

6. De Araujo FF, Di Bella ZIKDJ. Anticoncepção e planejamento

\section{CONCLUSÃO}

O uso de métodos de emergência é bastante frequente, o que pode acarretar gestações não planejadas. Apesar de parte das entrevistadas usarem fontes seguras de informação sobre sexualidade, isso não se reflete no uso consistente e informado de métodos contraceptivos. Mesmo com o conhecimento e informação, essa população ainda está exposta a situações de risco, como infecções sexualmente transmissíveis, uso inadequado de anticoncepcionais e gravidez indesejada. São necessárias novas estratégias para abordar a população adolescente com relação à vida sexual.

familiar. São Paulo: Atheneu; 2015. v.4.

7. Foresti RGR. Gravidez na adolescência: um estudo exploratório sobre o inicio da experiência da gravidez [dissertação]. São Paulo: Universidade Federal de São Paulo, Escola Paulista de Medicina, Departamento de Psiquiatria; 2001. p.1. Disponível em: http://www.scielo.br/sci66ggelo. php?script=sci_arttext\&pid=S1414-32832001000200017.

8. Almeida MCC, Aquino EML, Gaffikin L, Magnani RJ. Uso de contracepção por adolescentes de escolas públicas na Bahia. Rev Saude Publica. 2003;37(5):566-75. Disponível em: http:// www.revistas.usp.br/rsp/article/viewFile/31630/33516.

9. Brasil. Ministério da Saúde. Pesquisa nacional de demografia e saúde da criança de da mulher, dimensões do processo reprodutivo e da saúde da criança. Brasília; 2009. Disponível em: http://bvsms.saude.gov.br/bvs/publicacoes/pnds_crianca mulher.pdf.

10. HercowitzA. Gravidezna adolescência. Rev Pediatria Moderna. 2002;38(8):392-5. Disponível em: http://www.moreirajr.com. $\mathrm{br} /$ revistas.asp?id_materia $=2064 \&$ fase $=$ imprime.

11. Berek JS, editor. Tratado de ginecologia. 15a ed. Rio de Janeiro: Guanabara Koogan; 2014. 